\title{
Comparison of Ultrasonography with Magnetic Resonance Imaging for the Diagnosis of Liver Steatosis
}

Madiha M.Qureshi ${ }^{1 *}$, Hassan Saleem ${ }^{2}$, Raham Bacha ${ }^{3}$, Rustam A. Shah ${ }^{4}$

${ }^{1}$ Bachelors in Radiology, Shifa Tameer-e-Millat University, Islamabad, Masters of Science in Medical Ultrasound, the University of Lahore, Lahore, Pakistan

${ }^{2}$ MBBS, FCPS, FRCR Radiology, The Royal College of Radiologists, UK, College of Physicians \& Surgeons Pakistan, Karachi, Islamabad Diagnostic Centre, Islamabad, Pakistan

${ }^{3} \mathrm{MD}, \mathrm{PhD}$ Ultrasound Scholar, the University of Lahore, Lahore, Pakistan

${ }^{4}$ MBBS, FCPS Radiology, Shifa International Hospital, Islamabad, College of Physicians \& Surgeons Pakistan, Karachi, Islamabad Diagnostic Centre, Islamabad, Pakistan

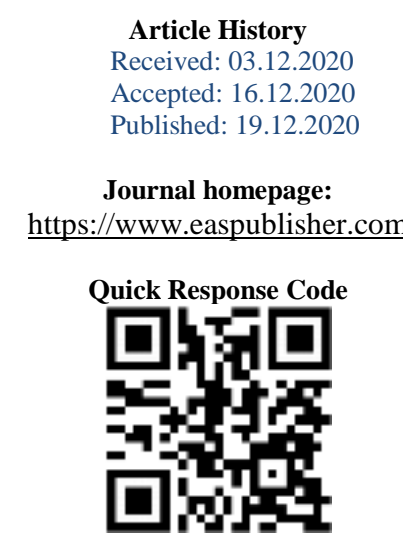

Abstract: Background: Nonalcoholic fatty liver disease ranges from simple steatosis, steatohepatitis - to advanced fibrosis and cirrhosis. It has great significance in medical practise as an increasingly recognized condition globally. Early detection and quantification to prevent its sequelae is necessary. Aims: We are keen to investigate minimally invasive technique to bring for the clinicians, a socio-economic issue of importance to the field of Radiology. Objective: To compare ultrasound with MRI for the diagnosis of liver steatosis. Methods: A prospective study was carried out at Radiology Department, IDC. Study duration: November 2018 - May 2019. 86 participants, aged 14-86 years, of either gender, with suspicion of steatosis were selected, after satisfying inclusion and exclusion criteria. Steatosis was confirmed utilizing Complex-based technique with MRI. For ultrasound, conventional sonographic signs criterion was used. Results: 88 consecutive patients, (43 females and 45 males) mean age (48.88 years). MRI-PDFF revealed steatosis grade 0 (normal) in $40.9 \%$ cases, $35.2 \%, 18.2 \%$ and $5.7 \%$ in grade 1,2 and 3 accordingly. While ultrasound revealed grade 0 in $26.1 \%$ cases and $44.3 \%, 25 \%$ and $4.5 \%$ in each respective grade. Sensitivity of ultrasound amplified as amount of hepatic fat gradually increased, from $58.33 \%$ (minimal steatotic cases) to $93.75 \%$ and $100 \%$ respectively (moderate to severe cases). Specificity remained between $73.68 \%$ to $98.82 \%$ throughout all cases. Conclusion: Ultrasound correlated well with MRI in moderate to severe hepatic steatosis, with mild degrees its specificity is reduced owing to other causes of liver injuries. Subjects practising unhealthy diet, hypertensive plus diabetics are more prone to develop steatosis. Thus its an acceptable modality for steatosis diagnosis, if severity/grading is neglected.

Keywords: Ultrasound, MRI, hepatic steatosis, NAFLD, noninvasive techniques, fatty liver. Abbreviations: HS (hepatic steatosis), NAFLD (non-alcoholic fatty liver disease), NASH (non-alcoholic steatohepatitis), PDFF (proton density fat fraction), US (ultrasound), USG (ultrasonography), LB (liver biopsy)

Copyright (C) 2020 The Author(s): This is an open-access article distributed under the terms of the Creative Commons Attribution 4.0 International License (CC BY-NC 4.0) which permits unrestricted use, distribution, and reproduction in any medium for non-commercial use provided the original author and source are credited.

\section{INTRODUCTION}

Hepatic or liver steatosis (HS) is a malady which is branded by accumulation of fats (usually, predominantly triglycerides) in a fraction of $5-10 \%$ of the whole liver mass. It is a histologic hallmartk of NAFLD. Its frequency is seemed to be more common in individuals who consume alcohol (Alcoholic Liver Disease - ALD) consistently but is also seen in those subjects which are non-consumers (Nonalcoholic fatty Liver Disease - NAFLD). In the latter case, the reason may be an assortment of issues, e.g; diabetes mellitus, obesity, hyperlipidemia are often co-existing conditions but choline deficiency, extended fasting, Wilson's disease, tirosinemia, total parenteral nutrition, malnutrition and sudden loss of a person's weight) are metabolism-related. Various surgical interventions (relating to gallbladder and pancreas, sleeve gastrectomy, gastroplasty for morbidly obese person, jejunoileal bypass surgery), certain medications (i.e; amiodarone, glucocorticoids, cytostatics etc) or toxins (like arsenic, $\mathrm{CCl} 4$ ) and mushrooms, frizzy drinks were investigated being responsible) [1].

The epidemiology and demographic characteristics of NAFLD vary greatly [2]. A probable prevalence of $25-35 \%$ is seen in overall populace of the U.S [3]. A study from Greece revealed evidence of steatosis in $31 \%$ of autopsied cases [4]. While in Asian states, there is a wide variation, from $5-40 \%$ [5]. It is note-worthy that its prevalence depends partly upon the 
methodology used to diagnose it, population heterogeneity and referral bias has also an impact on it, thatswhy different studies reveal different incidence rates [5]. So we considered prevalence as 7\%, according to number of daily occuring cases at our centre. From the past few decades, it was thought to be rare (maybe owing to deficiency of researches) in Asia. It establishes at a reduced BMI in Asian kingdoms and numerous subjects do not present with glucose intolerance $[6,7]$.

Nonalcoholic steatohepatitis (NASH) - the more advanced face of NAFLD, is always believed to be a potential risk factor for advancement towards cirrhosis [8]. NASH-related cirrhosis has developed as the second foremost indication for liver transplantations worldwide, as these patients at more risk for developing HCC [9]. Due to these serious indications, its earlier diagnosis is vital and pharmacological therapy for NASH is promptly required [10]. The specific pathogenesis of NAFLD remains little known. Yet it is believed to happen from deminished disposal of unsaturated fats due to debilitated mitochondrial $\beta$ oxidation. Toxins of certain bacteria, high production of cytokines (particularly tumor necrosis factor- $\alpha$ ) are likewise believed as potential triggers for progression from steatosis to NASH [11-13].

The traditional LB (liver biopsy), which was gold standard, has gained lesser value. Current investigations recommend that PDFF derived by MRI and MR-spectroscopy can really prove as a superior standard for reference for the evauation of HS than histologic assessment [9]. The reason behind is, these MR stratergies have proved to be highly accurate and reproducible [14]. It empowers exact, repeatable and reproducible evaluation of steatosis for the whole liver non-invasively. Histologic assessment is prone to great interobserver variability and sampling error [15]. This is not the case with NAFLD only, while performing LB, sampling variability is seen among other cases as well and there exists novel investigations cautioning about chances of experiencing sampling mistake in a vast range of viral as well as cholestatic diseases of liver [16].

The motivation behind why we favoured MRI over MRS lies in the fact that from a practical viewpoint, MRI seems to be superior compared with MRS. Acquisition and examination of MRS technique demands proficiency and is lengthy. The Single-voxel MRS technique, gathers information from a minute area of hepatic parenchyma (less than a voxel $\leq 3 \mathrm{~cm} \times 3 \mathrm{~cm}$ $x 3 \mathrm{~cm}$ ), that might be liable to sampling error, despite it is a lot bigger than a biopsy sample [17]. Since MRSPDFF and MRI-PDFF agree closely [18], the latter one is commonly preferred in clinical studies because of its more practicality and lesser sampling variability.
C. Isabela and colleagues, suggessted in a recent ancillary study that NASH represents a minor proportion compared to NAFLD and suggessted that non-invasive techniques with clinical information are sufficient for maximum cases of steatosis evaluation [19]. In another ancillary study, conducted by Hernaez and co-workers, proved sensitivity and specificity of sonography to be $84.8 \%$ and $93.6 \%$ respectively for steatotic liver assessment. They suggessted ultrasound to be imaging modality of preference in clinical setups and research trials [20].

Even though, MRI-PDFF is now used as a biomarker of $\mathrm{HS}$, the feasibility of utilizing this technique in research and clinical settings is restricted by the great cost and limited availibility, like the installation of PDFF software is still required with vast specifications in the MR-systems, which most of the clinical-setups lack in the country and also an undenieable fact of claustrophobic nature of a significant number of personages. Ultrasound, in contrast, is inexpensive, widely available and commonly used in all settings, including research and clinical practise, regardless of being less accurate and less precise than MRI-PDFF [8, 20]. Pathologists approve that little amount of steatosis with under $5 \%$ of hepatocytes involved, is insignificant and could not be regarded as a true pathologic anomaly [3]. So, its a worthwhile tool to screen fatty liver - defined as moderate to severe steatosis. It can be repeated in evolving monitoring because of no bio-effects. That's the reason ultrasound is utilized as priority for diagnosis. Recent trials in adult and children have shown that vitamin E (an anti-oxidant) could help improve NASH in non-diabetic patients [21]. Some studies recommend to maintain a healthy diet, which would have benefit beyond weight reduction for all NAFLD patients with and without obesity [22].

This research would be beneficial for those patients who are contraindicated to undergo MR-scan. It would convey how and in which cases sonography could really serve to rule-out hepatic steatosis cases efficiently. Children, intra-departmental \& old-aged patients would benefit in this regard as to cooperate in breath-hold sessions is nearly impossible for them. Motion artifact is the biggest disadvantage with MRI, which makes it a limited study. So Sonogram would serve as a second modality of choice, being bed-side available, no preparations and cautions. By comparing it with MRI, we can set clear-cut criteria for diagnosing steatosis using ultrasound, with relatively less scan time and decide which patients should undergo further testing. It could aid in surgical planning. Ultrasound can provide non-invasive prediction of liver histology [23]. Therefore it is intended to study comparison of Ultrasonography with Magnetic Resonance Imaging for the diagnosis of liver steatosis. 
Various USG signs of HS have been elaborated; however, to date, they have not been meticulously compared to MRI-PDFF. Therefore, the purpose of our study was to correlate conventional sonographic signs/grades and to assess its diagnostic accuracy to MRI-PDFF to forecast the existence and degree of HS.

\section{Materials ANd Methods \\ Study Design and Subjects}

This was a single-center, cross-sectional, prospective, observational clinical study of individuals, known to have or suspected of having NAFLD. It was conducted at Department of Diagnostic Radiology at Islamabad Diagnostic Centre, Islamabad, Pakistan. Study duration was six months, after ethical approval of synopsis from Institutional Review Board at The University of Lahore ( $1^{\text {st }}$ November $2018-1^{\text {st }}$ May 2019). All rules and regulations set by them were followed. Written informed consent was duly signed by each participant and their rights were respected. All information and data were kept confidential and participants remained anonymous throughout the study. The authors claimed no conflict of interests. Between Nov.2018 and May 2019, 88 consecutive patients, aged 14 to 86 years, among which $48.9 \%$ (43 of 88) were females (mean age, 53.86 years; 17.6 [standard deviation]; range, 15-87 years) and $51.1 \%$ (45 of 88) were males (mean age, 43.97 years; 18.8 [standard deviation]; range, 14-81 years) with suspicion of NAFLD, who underwent PDFF measurement as well as ultrasonography scans, in the same day, in the same setting (to quantify and stage the presence of hepatic steatosis) were included in this study. The subjects presenting with hepatorenal disease or absent right kidney (05 cases), those undergoing treatment with hepatotoxic, nephrotoxic, corticosteroid or immunosuppressant drugs were excluded from the study (08 cases), as well as those presenting with other chronic diseases of liver and primary or secondary hepatic malignancy (14 cases), also those who were pregnant or trying to become pregnant (02 cases) \& with contraindication to MRI and not agreeing to undergo USG in te same day (03 cases). The participants were submitted to ultrasound and MRI departments, laboratory tests and anthropometric evaluation. Trained nurses took medical history and health-related behaviours of all participants, using a standardised questionnaire. Existing use of medications was also taken into consideration.

\section{Anthropometric Measurements}

Biochemical, dietary, anthropometric, physical and standardized history data were accessed along with aforementioned scans to investigate the aims of this study. Body mass index (BMI) was calculated as body weight in kilograms divided by height in meters $(\mathrm{kg} / \mathrm{m} 2)$. Definitions of obesity were based on criteria from the World Health Organization and BMI from 25 to $29.9 \mathrm{~kg} / \mathrm{m} 2$ was considered overweight and BMI of $30 \mathrm{~kg} / \mathrm{m} 2$ or greater was considered to be obese [24].

\section{Imaging}

Subjects were instructed to fast for at least 5 hours before the scans. US and MRI scans were performed upon the same day, in the same settings.

\section{Equipment and Scanning Technique Ultrasound Machine}

Entire USG investigations were carried out employing a standardized scanning protocol on a Philips iU22 (Philips Healthcare, Andover, MA, USA) scanner with a low frequency convex transducer. Two radiologists (Dr. R.A \& Dr. F.J), who had 5 and 6 years of relevant practice, evaluated the USG images, using a picture archiving and communication system (PiView, Infinitt Co., Seoul, Korea). The clinical and MRI data was masked from them in advance. The observers utilized 2-point scale to detect the existence of HS: (1) steatosis (0) no steatosis. Any differing of opinion was resolved at the same time. Anyhow we didn't come across any.

\section{USG Signs and Diagnosis}

All sonographic signs (abnormal hepatorenal echoes, echogenicity loss of the portal vein, reduced diaphragm visualization and impaired visualisation of hepatic parenchyma) were assessed to detect and ascertain the severity of fatty liver, as done by one of previous studies [25]. On sonogram, an abnormal hepatorenal echo was recognized when the liver possessed greater echogenicity as compared to right renal-cortex and regarded as Grade 1 (Fig 2a). Echogenicity loss of portal vein was highlighted when main portal vein's echogenic wall was invisible in the right intercostal view and regarded as Grade 2 (Fig 2b). Impaired visualisation of hepatic parenchyma was demonstrated by impaired visualisation of over $1 / 3^{\text {rd }}$ of the hepatic parenchyma and poor diaphragm visualization was demarcated as compromised visualization of beyond half of the diaphragm in the right intercostal view and regarded as Grade 3 (Fig 2c).

The ultrasound scan for the measurement of liver was performed with the patient in supine position. The probe was held longitudinally and placed perpendicular to the examination table, upon which the patient lies. Looked in transverse up and down the left lobe from a subcostal approach. Observed in transverse through the right lobe subcostally or intercostally. Roll the patient in a left lateral decubitus position for assessment of right lobe. To measure the size of the liver, used a sagittal approach in the mid clavicular line.

\section{MR Scanner}

Hepatic MRI was performed using a $1.5 \mathrm{~T}$ magnet (Signa HDxt 1.5; GE Healthcare, Waukesha, Wis) equipped with a phased-array body coil, The protocol included a non-contrast MR-sequence software 
product (IDEAL-IQ; GE Healthcare), which is a threedimensional volumetric imaging sequence used to create $\mathrm{T}_{2} *$ and triglyceride fat fraction (FF) maps from a single breath-hold acquisition, as described by $\mathrm{Yu}$ et al., [26]. The technique was used to estimate R2* $(1 / \mathrm{T} 2 *)$ and PDFF (water-triglyceride fat separation) in the liver in a single simultaneous acquisition. Imaging involved a low flip angle (to suppress $\mathrm{T} 1$ effects) and multiple echo times (to estimate $\mathrm{T} 2 *$ effects); imaging FF was calculated by using Multiecho Analysis Method. The IDEAL (iterative decomposition of water and fat with echo asymmetry and least-squares estimation) method is a chemical-shift-based, water-fat separation method using both magnitude and phase information. To separate water and fat signals, this technique measures the local field map and demodulates it from the signal in the source images, using three or more echoes at different TEs. Although technically complex, the use of phase information for the IDEAL method allows PDFF to be measured over a full dynamic range of $0 \%-100 \%$ hepatic steatosis. Following its initial development, the algorithms for reducing $\mathrm{T} 1$ and noise-related bias, for $\mathrm{T} 2 *$-correction and for spectral modeling of fat, were implemented with the IDEAL method, allowing for T1- independent, $\mathrm{T} 2 *$-corrected estimation of PDFF, as done by previos studies [18].

Subjects were scanned supine with a torso phased-array coil, centered over the abdomen, at the level of the liver, supported with belts, ensuring no motion of any body parts. Emergency bell kept in one hand of patient. Scan ran for approximately 18 seconds. Other imaging parameters were 8 -mm slice thickness, 20 recorded slices, 0\% interslice gap, one signal average and rectangular field-of-view adjusted to individual body habitus and breath-hold capacity.

\section{Measurement of MRI PDFF}

MR images were transferred to a Picture Archiving and Communication System (PACS) workstation for analysis. Blinded to USG results, a trained research fellow (6 years of experience) manually placed circular regions of interest (ROIs) in each of the nine Couinaud liver segments on the MR imaging PDFF maps in each subject. Each ROI had a radius of $1 \mathrm{~cm}$ approx. or $100-\mathrm{mm} 2$ and was placed near the center of each segment, while avoiding major vessels, ducts, organ boundaries, focal hepatic lesions and imaging artifacts. The PDFF in each of the nine singular, non-overlapping ROIs was recorded across the entire liver and the average of all measurements was defined as the mean PDFF, as performed by Tang et al. and Mimi Kim et al., [27].

\section{Reference Standard of Fatty Liver}

Fatty liver was graded according to the following criterion: Grade 0: PDFF less than 6.4\%, Grade 1: PDFF equal to or greater than $6.4 \%$ and less than $17.4 \%$, Grade 2: PDFF equal to or greater than
$17.4 \%$ and less than $22.1 \%$, and Grade 3: PDFF equal to or greater than $22.1 \%$. These thresholds were taken from the study done by Tang et al., [28].

\section{Statistical ANALYSIS}

Statistical analysis was performed by a biostatistical analyst (M.F., with 10 years of experience) working under the supervision of a faculty biostatistician (A.H., with more than 15 years of experience) with statistical computig software (R version 2.15.1; $\mathrm{R}$ Foundation for statistical computing, Vienna, Austria). Subjects'demographic, anthropometric and imaging information was summarized descriptively. Categorical variables were expressed as numbers and percentages and continuous variables were described as means \pm standard deviations([SD]). MRI PDFF of each USG sign were calculated. The sensitivity, specificity, positive predictive value (PPV), negative predictive value (NPV) with $95 \%$ confidence intervals were calculated, using Wilson's score to evaluate the ability of USG signs to predict the degree of HS.

$\mathrm{p}<0.05$ was considered statistically significant. We used SPSS Version 25.0 (SPSS Chicago, IL, USA) and Medcalc for Windows (Version 14.12.0; MedCalc software, Mariakerke, Belgium) for statistical analyses.

\section{Calculation of Sample size}

For the purpose of sample size calculation, data from previous studies was taken into consideration, suggessting different numbers, according to different regions \& ethnicities. So, an estimate from previous data and occurrence of local number of cases, incidence was found to be $7 \%$ and calculated sample size is 86 [5, 29].

\section{Results}

A total of 88 patients were included in the study. Among them $48.9 \%$ (43 of 88 ) were females and $51.1 \%$ (45 of 88 ) were males. Mean age was 48.81 (SD \pm 18 ) and mean BMI was 27.59 (SD \pm 4.8$)$. Other anthropometric measurements are presented in Table-1.

Mean MRI-PDFF was calculated to be 8.95 $(\mathrm{SD} \pm 4.80)$. MRI-PDFF revealed hepatic steatosis in $59 \%$ of subjects (52/88). Among them $46.15 \%$ (24/52) were males and $53.85 \%(28 / 52)$ were females. While ultrasonography identified steatosis in $73.8 \%$ (65/88). In a subtotal of 88 cases, 50 were the cases in which both modalities proved steatosis, while both agreed to normal liver cases were 21 (discrepancy cases of normal liver cases were 15 only). In subjects with no sonographic sign, PDFF revealed to be $2.79 \%$ (SD \pm 0.79). While in subjects with all the sonographic signs positive, PDFF was $24.27 \%$ (SD \pm 6.29 ). Fig 1 (a,b,c) shows MRI scans of subjects with grade 1,2 and 3 respectively. 
MRI-PDFF revealed steatosis grade 0 (normal) in $40.9 \%(36 / 88)$ of cases, males were $(24 / 36)$ and females were (15/36). Grade 1 (mild) in $35.2 \%(31 / 88)$ cases, males were (12/31) and females were (17/31). Grade 2 (moderate) in $18.2 \%(16 / 88)$ cases, males $(07 / 16)$ and females $(09 / 16)$. Grade 3 (severe) in $5.7 \%$ $(05 / 88)$ of subjects, including males $(02 / 05)$ and females $(03 / 05)$. While ultrasound revealed grade 0 in $26.1 \%$ of cases, grade 1 in $44.3 \%$, grade 2 in $25 \%$ and grade 3 in $4.5 \%$ of patients.

For Grade 0 cases, sensitivity and specificity of ultrasound were $58.33 \%$ and $96.15 \%$ respectively. Diagnostic Accuracy driven out to be $80.68 \%$. For Grade 1 cases, sensitivity and specificity were $77.42 \%$ and $73.68 \%$. Diagnostic Accuracy was $75 \%$. For Grade 2 cases, sensitivity was $93.75 \%$ and specificity was 90.28\%. While Diagnostic Accuracy found to be $90.91 \%$. For Grade 3 cases, sensitivity rose to $100 \%$, seemingly specificity arose to $98.82 \%$. In this case, Diagnostic Accuracy turned out to be $98.86 \%$. Sensitivity of ultrasound amplified as the amount of hepatic fat gradually increased, from $58.33 \%$ (in minor amounts of fat) to $93.75 \%$ and $100 \%$ respectively (in moderate to severe amounts of fats). While specificity remained between $73.68 \%$ to $98.82 \%$ throughout all cases of steatosis.

In subjects with grade 1 steatosis, diabetes was sequentially found to be present in utmost cases. Same is the case with hypertension. We had $<7 \%$ cases with chemo/radiation therapy and CAD, that's why we are unable to comment on this aspect. But those minor cases were found to be positive for HS and also some previous studies exposed them to be responsible [30, 31]. Hyperlipidemia was at 'mildly high' degree among positive steatotic cases; $23 / 52(44.23 \%)$ and was not significantly observed among subjects with grade 1 steatosis $08 / 31(25.8 \%)$. AST/ALT ratio was variable among different grades. Yet it reveals from our results that this ratio may or may not be high among patients with minimal to mild hepatic steatosis. All of this data is illustrated in Table-1.

\section{Baseline Characteristics}

Table-1: Baseline characteristics according to degree of fatty liver. Data is presented as mean with standard deviation or as number of subjects with percentage in paranthesis

\begin{tabular}{|c|c|c|c|c|c|c|}
\hline \multirow[t]{2}{*}{ Variables (Mean) } & Normal & Grade 1 & Grade 2 & Grade 3 & \multirow{2}{*}{$\begin{array}{l}\begin{array}{l}P \text { - } \\
\text { value }\end{array} \\
-\end{array}$} & \multirow{2}{*}{$\begin{array}{l}\text { P-value } \\
\text { for trend } \\
-\end{array}$} \\
\hline & $\begin{array}{l}\text { PDFF } \\
6.4 \%)\end{array}$ & $\begin{array}{l}(6.4 \% \leq \text { PDFF }< \\
16.3 \%)\end{array}$ & $\begin{array}{l}(16.3 \% \leq \text { PDFF } \\
21.7 \%)\end{array}$ & $\begin{array}{ll}\text { (PDFF } & \geq \\
21.7 \%) & \end{array}$ & & \\
\hline $\mathrm{N}(\%)$ & 36 & 31 & 16 & 5 & - & - \\
\hline Mean PDFF (\%) & 5.63 & 7.50 & 15.94 & 26.62 & - & \\
\hline Age (year) & $45.2 \pm 19.5$ & $50.3 \pm 18.4$ & $52.8 \pm 19.3$ & $34.6 \pm 22.2$ & $<0.01$ & $<0.01$ \\
\hline $\mathrm{M}: \mathrm{F}$ & $41: 15$ & $14: 17$ & $7: 9$ & $3: 2$ & - & - \\
\hline BMI & $25.5 \pm 4.24$ & $28.3 \pm 3.89$ & $30.55 \pm 5.79$ & $28.46 \pm 4.96$ & $<0.01$ & $<0.01$ \\
\hline $\begin{array}{l}\text { Hyperlipidemia } \\
\text { (pr,ab) }\end{array}$ & $\begin{array}{l}\mathrm{pr}=6 \\
\mathrm{ab}=30\end{array}$ & $\begin{array}{l}\mathrm{pr}=8 \\
\mathrm{ab}=23\end{array}$ & $\begin{array}{l}\mathrm{pr}=12 \\
\mathrm{ab}=4\end{array}$ & $\begin{array}{l}\mathrm{pr}=3 \\
\mathrm{ab}=2\end{array}$ & $<0.01$ & $<0.01$ \\
\hline AST/ALT ratio & $0.81 \pm 0.04$ & $0.8 \pm 0.07$ & $1.05 \pm 0.09$ & $1.12 \pm 0.17$ & $<0.05$ & $<0.05$ \\
\hline Diabetes (pr,ab) & $\begin{array}{l}\mathrm{pr}=7 \\
\mathrm{ab}=29\end{array}$ & $\begin{array}{l}\mathrm{pr}=12 \\
\mathrm{ab}=19\end{array}$ & $\begin{array}{l}\mathrm{pr}=2 \\
\mathrm{ab}=14\end{array}$ & $\begin{array}{l}\mathrm{pr}=1 \\
\mathrm{ab}=4\end{array}$ & $<0.25$ & $<0.05$ \\
\hline HTN (pr,ab) & $\begin{array}{l}\mathrm{pr}=12 \\
\mathrm{ab}=24\end{array}$ & $\begin{array}{l}\mathrm{pr}=13 \\
\mathrm{ab}=18\end{array}$ & $\begin{array}{l}\mathrm{pr}=3 \\
\mathrm{ab}=13\end{array}$ & $\begin{array}{l}\mathrm{pr}=2 \\
\mathrm{ab}=3\end{array}$ & $<0.05$ & $<0.05$ \\
\hline C.A disease (pr,ab) & $\begin{array}{l}\mathrm{pr}=3 \\
\mathrm{ab}=33\end{array}$ & $\begin{array}{l}\mathrm{pr}=2 \\
\mathrm{ab}=29\end{array}$ & $\begin{array}{l}\mathrm{pr}=1 \\
\mathrm{ab}=15\end{array}$ & $\begin{array}{l}\mathrm{pr}=0 \\
\mathrm{ab}=5\end{array}$ & $<0.05$ & $<0.05$ \\
\hline Body habitus & $\begin{array}{l}\text { nor }=19 \\
\text { o.w }=12 \\
\text { ob }=5\end{array}$ & $\begin{array}{l}\text { nor }=6 \\
\text { o.w }=13 \\
\text { ob }=12\end{array}$ & $\begin{array}{l}\text { nor }=3 \\
\text { o.w }=5 \\
\text { ob }=8\end{array}$ & $\begin{array}{l}\text { nor }=0 \\
\text { o.w }=3 \\
\text { ob }=2\end{array}$ & $<0.05$ & $<0.05$ \\
\hline $\begin{array}{l}\text { Unhealthy diet (y, } \\
\text { no) }\end{array}$ & $\begin{array}{l}y=1 \\
\text { no }=35\end{array}$ & $\begin{array}{l}y=1 \\
\text { no }=30\end{array}$ & $\begin{array}{l}y=4 \\
\text { no }=12\end{array}$ & $\begin{array}{l}y=1 \\
\text { no }=4\end{array}$ & $<0.01$ & $<0.01$ \\
\hline Surgical hx.(pr,ab) & $\begin{array}{l}\mathrm{pr}=26 \\
\mathrm{ab}=10\end{array}$ & $\begin{array}{l}\mathrm{pr}=14 \\
\mathrm{ab}=17\end{array}$ & $\begin{array}{l}\mathrm{pr}=10 \\
\mathrm{ab}=6\end{array}$ & $\begin{array}{l}\mathrm{pr}=2 \\
\mathrm{ab}=3\end{array}$ & $<0.05$ & $<0.05$ \\
\hline $\begin{array}{l}\text { Chemo/rad. } \\
\text { therapy }\end{array}$ & $\begin{array}{l}y=1 \\
\text { no }=35\end{array}$ & $\begin{array}{l}y=2 \\
\text { no }=29\end{array}$ & $\begin{array}{l}y=2 \\
\text { no }=14\end{array}$ & $\begin{array}{l}\mathrm{y}=0 \\
\text { no }=5\end{array}$ & $<0.05$ & $<0.05$ \\
\hline \multicolumn{7}{|l|}{ USG signs } \\
\hline Normal (Grade 0) & $21(58.3 \%)$ & 0 & 0 & 2 & - & $<0.01$ \\
\hline Grade 1 & 15 & $24(77.4 \%)$ & 0 & 0 & - & $<0.01$ \\
\hline Grade 2 & 0 & 7 & $15(93.8 \%)$ & 0 & - & $<0.01$ \\
\hline Grade 3 & 0 & 0 & 1 & $3(60 \%)$ & - & $<0.01$ \\
\hline
\end{tabular}

p < 0.05 by ANOVA. $p<0.01$ by Spearson correlation analysis; M:F, male:female; BMI, body-mass index; AST/ALT, aspartate aminotransferase/alanine transaminase; pr, present; ab, absent; HTN, hypertension; C.A disease, Coronary Artery disease; nor, normal; o.w, overweight; ob, obese; y, yes; hx, history; rad., radiation 
Table-2: Table showing ultrasound and MRI gradings cross-tabulation USG, ultrasonography; Diag, Diagnosis; MR, MRI

\begin{tabular}{|c|c|c|c|}
\hline \multicolumn{4}{|c|}{ Correlations } \\
\hline & & Body mass index & Mean MRI PDFF \\
\hline \multirow[t]{3}{*}{ Body mass index } & Pearson Correlation & 1 & .184 \\
\hline & Sig. (2-tailed) & & .086 \\
\hline & $\mathrm{N}$ & 88 & 88 \\
\hline \multirow[t]{3}{*}{ Mean MRI PDFF } & Pearson Correlation & .184 & 1 \\
\hline & Sig. (2-tailed) & .086 & \\
\hline & $\mathrm{N}$ & 88 & 88 \\
\hline
\end{tabular}

Table-3: Table showing correlation between BMI (body mass index) and Mean MRI PDFF

\begin{tabular}{|c|c|c|c|c|c|c|c|}
\hline \multicolumn{8}{|c|}{ USG Diag-Grading * MRI Diag-Grading Cross-tabulation } \\
\hline & & & \multicolumn{4}{|c|}{ MR Diag-Grading } & \multirow[t]{2}{*}{ Total } \\
\hline & & & 0 & 1 & 2 & 3 & \\
\hline \multirow[t]{8}{*}{ USG Diag-Grading } & \multirow[t]{2}{*}{0} & Count & 21 & 0 & 0 & 2 & 23 \\
\hline & & $\%$ within MR Diag-Grading & $53.8 \%$ & $0.0 \%$ & $0.0 \%$ & $40.0 \%$ & $26.1 \%$ \\
\hline & \multirow[t]{2}{*}{1} & Count & 15 & 24 & 0 & 0 & 39 \\
\hline & & \% within MR Diag-Grading & $41.7 \%$ & $82.7 \%$ & $0.0 \%$ & $0.0 \%$ & $44.3 \%$ \\
\hline & \multirow[t]{2}{*}{2} & Count & 0 & 7 & 15 & 0 & 22 \\
\hline & & $\%$ within MR Diag-Grading & $0.0 \%$ & $22.6 \%$ & $88.2 \%$ & $0.0 \%$ & $25.0 \%$ \\
\hline & \multirow[t]{2}{*}{3} & Count & 0 & 0 & 1 & 3 & 4 \\
\hline & & $\%$ within MR Diag-Grading & $0.0 \%$ & $0.0 \%$ & $6.3 \%$ & $60.0 \%$ & $4.5 \%$ \\
\hline \multirow{2}{*}{\multicolumn{2}{|c|}{ Total }} & Count & 36 & 31 & 16 & 5 & 88 \\
\hline & & \% within MR Diag-Grading & $100.0 \%$ & $100.0 \%$ & $100.0 \%$ & $100.0 \%$ & $100.0 \%$ \\
\hline
\end{tabular}

Table-4: Table showing steatosis cases positive on ultrasound and their relationship with AST/ALT ratio among participants

\begin{tabular}{|c|c|c|c|c|c|c|}
\hline 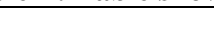 & USG & AST/ALT & AST/ALT ratio $=$ & AST/ALT & AST/ALT ratio & AST/ALT \\
\hline & $\begin{array}{r}\text { positive } \\
\text { cases }\end{array}$ & ratio $\begin{array}{c}= \\
\text { (normal) }\end{array}$ & $\begin{array}{rr}0.9 \quad \text { (minimally } \\
\text { elevated) }\end{array}$ & $\begin{array}{r}\text { ratio }=1.0 \\
\text { (elevated) }\end{array}$ & $\begin{array}{r}=1.1 \text { (slightly } \\
\text { high) }\end{array}$ & $\begin{array}{r}\text { ratio }=1.3 \\
\text { (high) }\end{array}$ \\
\hline $\mathrm{N}$ & 65 & 40 & 07 & 06 & 11 & 03 \\
\hline Grade 1 & 39 & 36 & 02 & 0 & 01 & 0 \\
\hline Grade 2 & 22 & 4 & 04 & 05 & 09 & 0 \\
\hline Grade 3 & 04 & 0 & 01 & 01 & 01 & 03 \\
\hline True positive & 26 & & & & & \\
\hline True negative & 19 & & & & & \\
\hline False positive & 3 & & & & & \\
\hline False negative & 25 & & & & & \\
\hline
\end{tabular}

$\mathrm{N}$, total number of positive cases; AST, aspartase aminotransferase; ALT, alanine transaminase

MRI \& Ultrasound Diagnosis - Grading Comparisons

Ultrasound Grade 0 with MRI Grade 0

Table-5: Table showing ultrasound results marking as Grade 0 \& their direct comparisons with MRI results categorized as Grade 0

\begin{tabular}{|r|r|r|r|}
\hline Parameter & Estimate & Lower - Upper (95\% Cis) & Method \\
\hline Sensitivity & $58.33 \%$ & $\left(42.2,72.86^{1}\right)$ & Wilson Score \\
\hline Specificity & $96.15 \%$ & $\left(87.02,98.4^{1}\right)$ & Wilson Score \\
\hline Positive Predictive Value & $91.3 \%$ & $\left(73.2,97.58^{1}\right)$ & Wilson Score \\
\hline Negative Predictive Value & $76.92 \%$ & $\left(65.36,85.49^{1}\right)$ & Wilson Score \\
\hline Diagnostic Accuracy & $80.68 \%$ & $\left(71.22,87.57^{1}\right)$ & Wilson Score \\
\hline
\end{tabular}

Gr, grade; Cis, confidence intervals; USG, ultrasonography

Ultrasound Grade 1 with MRI Grade 1

Table-6: Table showing ultrasound results marking as Grade $1 \&$ their direct comparisons with MRI results categorized as Grade 1

\begin{tabular}{|r|r|r|r|}
\hline Parameter & Estimate & Lower - Upper (95\% Cis) & Method \\
\hline Sensitivity & $77.42 \%$ & $\left(60.19,88.61^{\mathbf{1}}\right)$ & Wilson Score \\
\hline Specificity & $73.68 \%$ & $\left(61.02,83.35^{\mathbf{1}}\right)$ & Wilson Score \\
\hline Positive Predictive Value & $61.54 \%$ & $\left(45.9,75.1^{\mathbf{1}}\right)$ & Wilson Score \\
\hline Negative Predictive Value & $85.71 \%$ & $\left(73.33,92.9^{1}\right)$ & Wilson Score \\
\hline Diagnostic Accuracy & $75 \%$ & $\left(65.04,82.87^{1}\right)$ & Wilson Score \\
\hline
\end{tabular}




\section{Ultrasound Grade 2 with MRI Grade 2}

Table-7: Table showing ultrasound results marking as Grade 2 \& their direct comparisons with MRI results categorized as Grade 2

\begin{tabular}{|r|r|r|r|}
\hline Parameter & Estimate & $\begin{array}{r}\text { Lower - Upper } \\
(\mathbf{9 5 \%} \text { Cis) }\end{array}$ & Method \\
\hline Sensitivity & $93.75 \%$ & $\left(71.67,98.89^{1}\right)$ & Wilson Score \\
\hline Specificity & $90.28 \%$ & $\left(81.26,95.21^{1}\right)$ & Wilson Score \\
\hline Positive Predictive Value & $68.18 \%$ & $\left(47.32,83.64^{1}\right)$ & Wilson Score \\
\hline Negative Predictive Value & $98.48 \%$ & $\left(91.9,99.73^{1}\right)$ & Wilson Score \\
\hline Diagnostic Accuracy & $90.91 \%$ & $\left(83.07,95.32^{1}\right)$ & Wilson Score \\
\hline
\end{tabular}

Ultrasound Grade 3 with MRI Grade 3

Table-8: Table showing ultrasound results marking as Grade 3 \& their direct comparisons with MRI results categorized as Grade 3

\begin{tabular}{|l|l|l|l|}
\hline Parameter & Estimate & Lower - Upper (95\% Cis) & Method \\
\hline Sensitivity & $100 \%$ & $\left(43.85,100^{\mathbf{1}}\right)$ & Wilson Score \\
\hline Specificity & $98.82 \%$ & $\left(93.63,99.79^{\mathbf{1}}\right)$ & Wilson Score \\
\hline Positive Predictive Value & $75 \%$ & $\left(30.06,95.44^{\mathbf{1}}\right)$ & Wilson Score \\
\hline Negative Predictive Value & $100 \%$ & $\left(95.63,100^{\mathbf{1}}\right)$ & Wilson Score \\
\hline Diagnostic Accuracy & $98.86 \%$ & $\left(93.84,99.8^{\mathbf{1}}\right)$ & Wilson Score \\
\hline
\end{tabular}

\section{MRI Images}

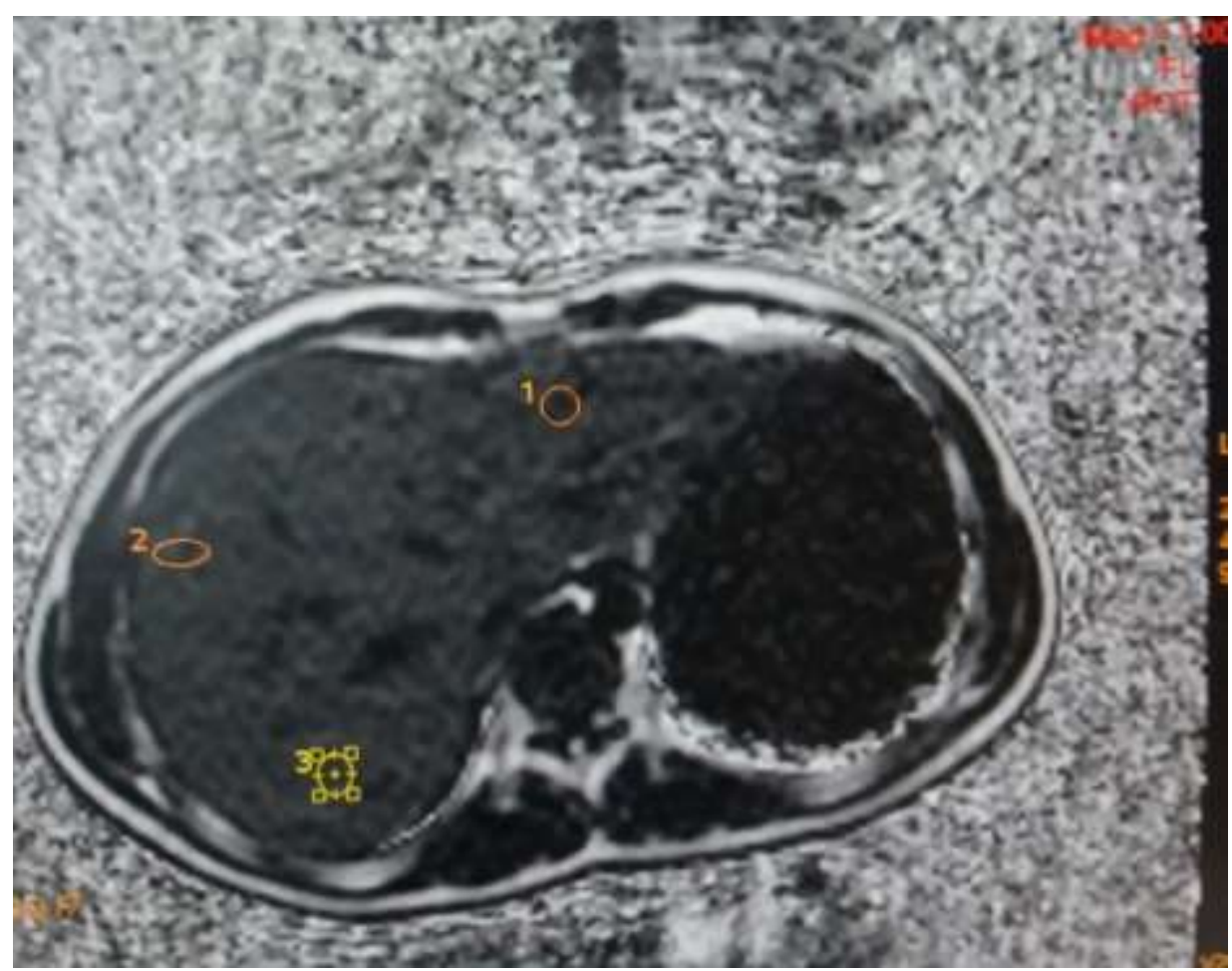

Fig-1a: Grade-1 (Fig showing MRI-PDFF scan of 81 years old, male participant, with grade 1 hepatic steatosis) 


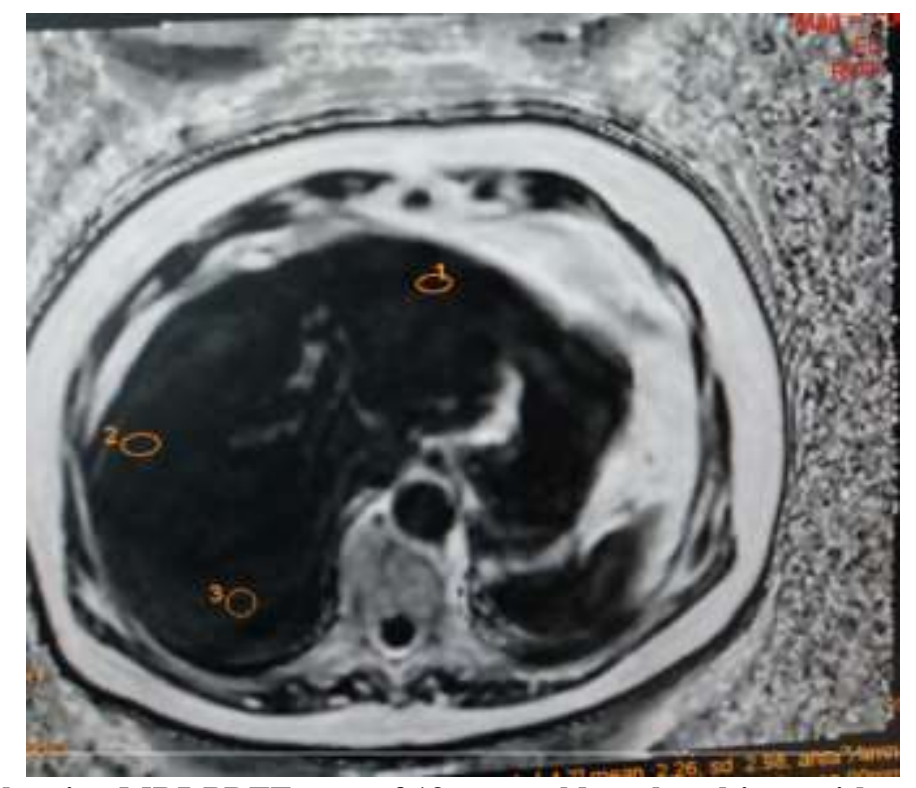

Fig-1b: Grade-2 (Fig showing MRI-PDFF scan of 19 years old, male subject, with grade 2 hepatic steatosis)

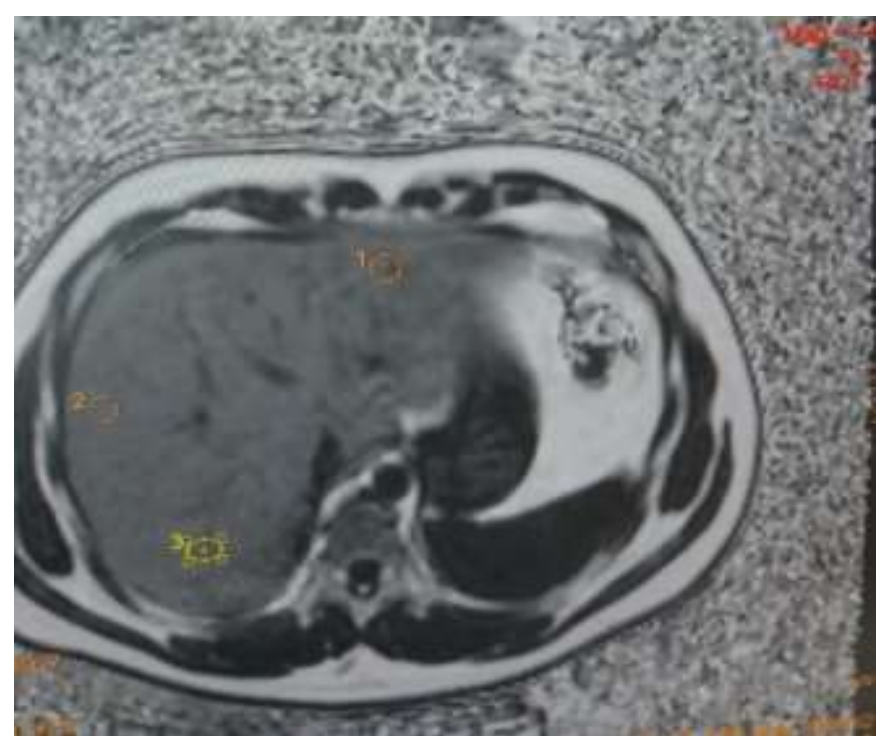

Fig-1c: Grade-3 (Fig showing MRI-PDFF of 29 years old, male subject, with grade 3 hepatic steatosis)

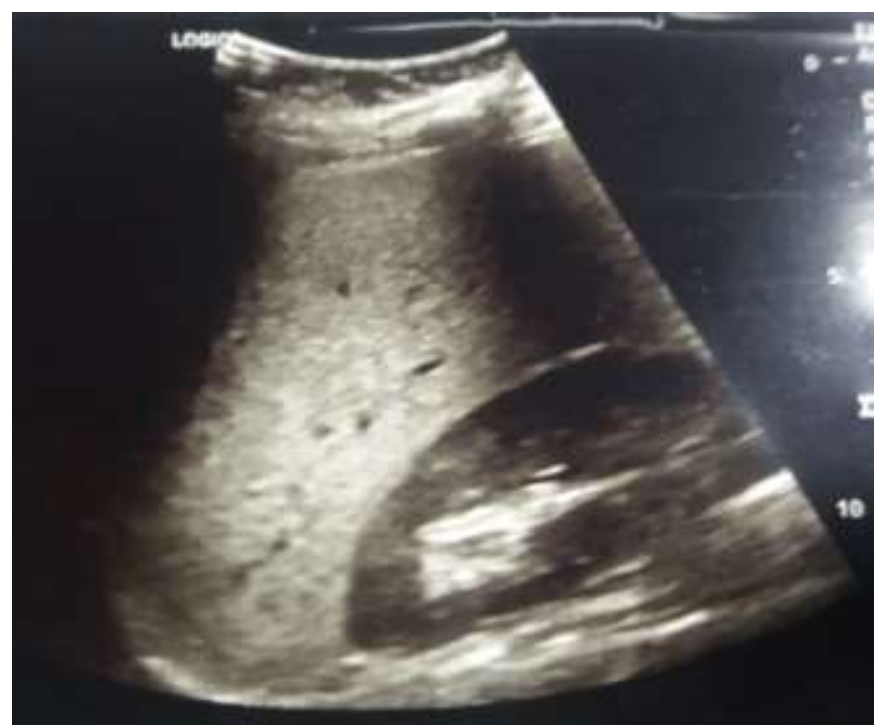

Fig-2a: Grade-1 (Fig showing ultrasound scan of 26 years old, female subject, with grade 1 hepatic steatosis) 


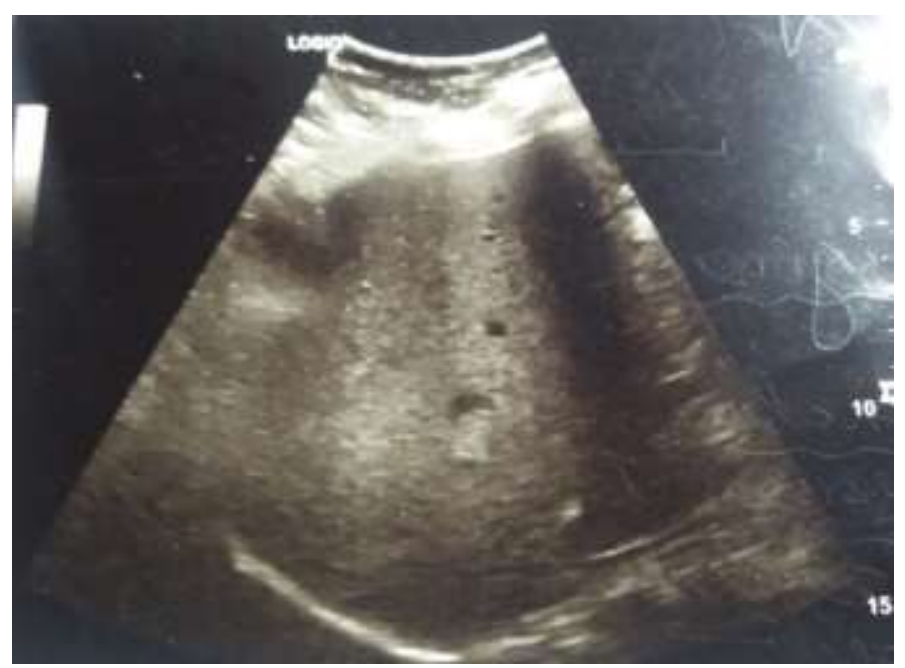

Fig-2b: Grade-2 (Fig showing ultrasound scan of 45 years old, female subject, with grade 2 hepatic steatosis)

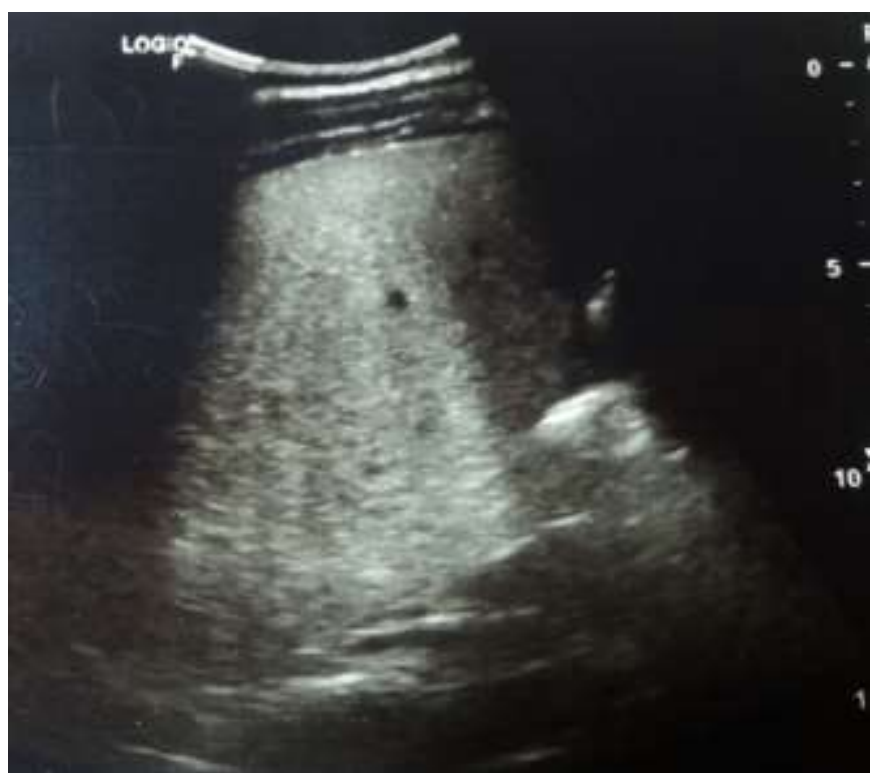

Fig-2c: Grade-3 (Fig showing ultrasound scan of 29 years old, male subject, with grade 3 hepatic steatosis)

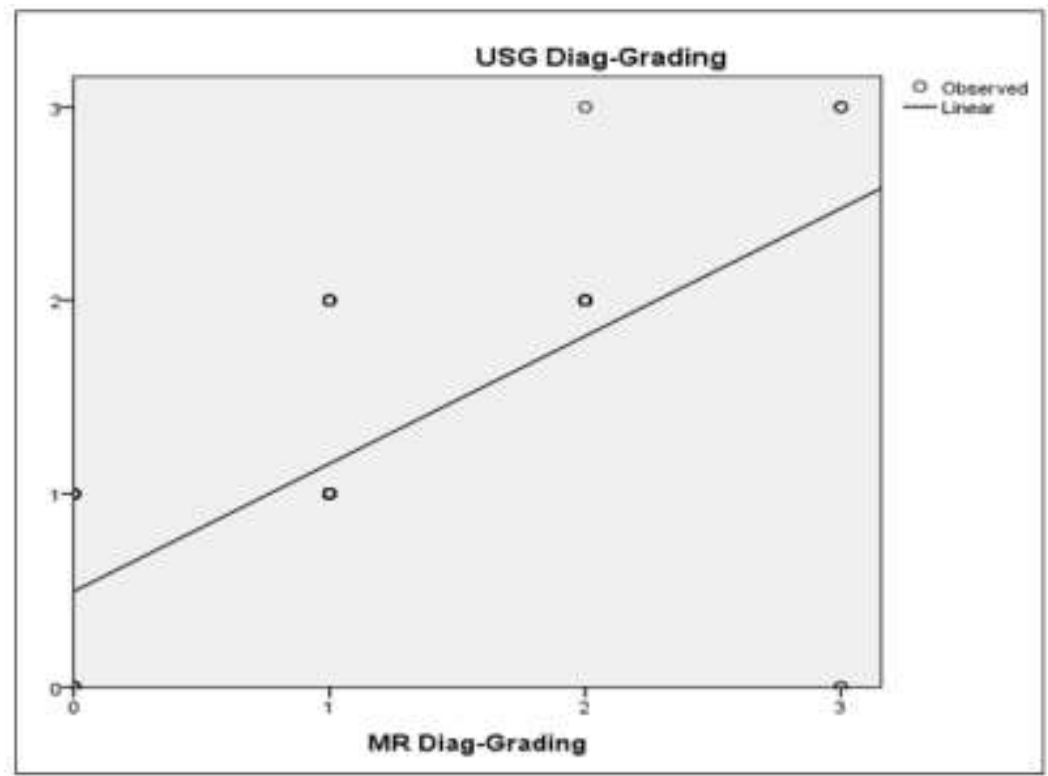

Fig-3a: A plot-graph illustrating relationship between MRI and Ultrasound diagnosis 


\section{DiscuSSION}

The current research was designed to determine the close correlation between MRI and ultrasound results, to diagnose hepatic steatosis efficiently through sonography, (while keeping MRI as reference standard). We ought to evaluate the longstanding prognostic significance of sonographic verdicts, along with its diagnostic implications in the field of Radiology, with some clinical examinations to rule out hepatic steatosis. We also tried to find possible causes of steatosis among Asians.

Ultrasound revealed excellent sensitivity as well as specificity for the sonographic assessment of NAFLD, while taking into account severe cases of steatosis (grade 2 and 3), while with mild cases (grade $0-1$ ) its sensitivity was reduced and this is the stage where MRI would be recommended. Specificity could be improved by addition of liver enzymes in investigational planning. Anyhow we recognize that for some clinical or research indications, high sensitivity may be preferred over high specificity.

Out of 5 severe HS cases, 2 were falsely positive to be grade 3 on MRI only due to iron overload, while they were normal upon US. Anyhow, this was not our topic of discussion and some previous studies torch lights this same aspect [32]. Some of the subjects with moderate to severe steatosis were investigated from our study to practise unhealthy diet (low quality oil/butter, frizzy drinks) in daily routine. Increased BMI and surgical histories (abdominal) were also discovered to be significant in development of HS in our participants. Increased BMI may or may not be observed in every subject affected from steatosis but most cases do. Previous studies reveal that individuals practising Keto diet, following weight-loss journey were affected significantly from steatosis.

My study's results are sufficiently comparable with the results of the research conducted by Mimi Kim et al., [27]. The purpose of his study was to correlate conventional USG signs to MRI PDFF. The prevalence of fatty liver (MRI PDFF $\geq 6.5 \%$ ) was $32.4 \%$ (59/182). There was a strong positive correlation between USG signs and MRI PDFF $(\sigma=0.780, \mathrm{p}<0.001)$ just like our study. They concluded that the sensitivity and NPV for the determination of HS by sonogram were good at $96.6 \%$ and $97.7 \%$ and USG may be considered a suitable screening tool for the exclusion of fatty liver. The difference with our study lies that they compared combinations of different sonographic signs alone and in combination to detect HS, while we made direct comparisons of each USG grade, with each relative MRI grades. We found this method more practical and suitable for our study.

Also, with the study conducted by M. L. Kromrey et al., [32]. Accuracy of ultrasonography was assessed to rule out hepatic steatosis, using MRI as reference standard. For different degrees of HS, sensitivity, specificity and accuracy of B-mode sonography were determined. MRI revealed HS in $40 \%$ of participants $(n=1,112)$, Sonography detected HS in $37.8 \%(n=1,052)$, corresponding to $74.5 \%$ sensitivity and $86.6 \%$ specificity. Their study concluded that ultrasonography is an excellent tool to assess HS in the clinical setting, with some limitations in patients with a low liver fat content. The difference with our study lies in the sample size of the study population, which was difficult to attain during a given span-time. Also they calculated R2* to detect liver iron-overload, we did the same to see haemochromatosis patients separately but we just gone through their values subjectively, as we were concerned with fat vacuoles only.

A study conducted by Bohte et al., [33] proved sensitivity and specificity to be $85.4 \%$ and $55.4 \%$ out of 104 participants, while carrying out MR spectroscopy and comparing it with ultrasound, for the detection of HS. MRI detected HS in $46.2 \%$ of subjects. They concluded that alone ultrasound is not able to accurately foresee the existence and severity of steatosis (more specifically) in severly obese adults and an additional utility of MRI is required [33]. As they utilized a different MRI technique for the diagnosis, hence cannot be compared directly with our results.

Utilizing ultrasonography, several clinical researches depicted sensitivities and specificities for diagnosing HS, ranging from 60-94\% [34]. But as different studies utilized different referene standards, direct comparisons would not be possible. Our study's results are different from other relevant literature in the way that we targeted Asian population specifically, while most of the studies conducted before included Western or other populations. Secondly, we performed both ultrasound and MRI scans in the same day, as well as in the same setting, as to avoid any differing of opinions. We also ruled out possible causes of steatosis in our study population, while other studies (in our best knowledge) only discussed its diagnosis from imaging modalities of preference.

Substantial hepatic steatosis can affect liver graft survival. If the degree of steatosis is $>30 \%$, there exists a $25 \%$ risk of evolving primary hepatic nonfunction. Thus, this mark of steatosis is a contraindication for liver transplantation. Liver biopsy is the existing reference standard for the evaluation of steatosis in Live liver donors. An accurate technique for the quantitative assessment of steatosis could spare healthy volunteers the necessity to undergo L.B. For this reason, quantitative ultrasound has been developed, which can characterize tissue microstructure objectively to as low as $5 \%$ of fats - the Computerized hepatorenal sonographic index. This innovative technique is an objective quantitative tool, operator-independent and overcomes the current known limitation of conventional sonography to subjectively diagnose steatosis [35]. 
Also, it is not influenced by fibrosis or in case of steatohepatitis.

The limitation of our study could be the sample size of study population. However, the complexity of methodology used, alongwith unique comparative diagnosis by both MRI-PDFF and Ultrasound in a short study duration of 6 months, made it critical to evaluate a large sample of patient population during the span of study. However a recent study, published in Feb 2019, by M. L. Kromrey et al., [32] included a much greater number of subjects and revealed a sensitivity of $74.5 \%$ and a specificity of $86.6 \%$ utilizing B-mode ultrasound and proved it a suitable imaging modality for diagnosing liver fat [32].

In order to rule out fatty profiles, with mild degrees on sonogram, one could recommend utilizing a combination of liver enzymes, followed by MRI scan, would be an appropriate approach. Also, we would like to make it a point to be highlighted, that researchers should follow and make one study as reference standard for MRI-cutoff points from now on. Its worth mentioning that patients with possible renal diseases, can be mistakenly regarded as grade 0 for $\mathrm{HS}$ on ultrasound, so RFT's should be cleared before the USG scan. Rigorous investigation should be carried out about clinical impact of "minimal to mild" hepatic steatosis. A recent study published in European Respiratory Journal 2019, elucit lung steatosis to be a confounder in asthma \& as a matter of fact, asthmatic patients are more prone to pneumonia. This can move our direction to ongoing COVID-19 pandemic, which attacks lung alveoli. This significant study was conducted by JG Elliot et al., [36]. Their data showed that adipose tissue is present within the airway wall. They suggessted that in an overweight person, deposition of adipose tissue may contribute to airway pathophysiology. They proposed another reason why maintaining a healthy weight is soo important to the proper functioning of human body [36]. Studies are needed to evaluate which groups are more badly affected by corona virus, taking into account age, BMI, previous respiratory diseases etc.

\section{CONCLUSION}

Ultrasound correlated well with the results of MRI and showed good diagnostic confidence, when there was present moderate to severe hepatic steatosis, while with mild degrees, its specificity is reduced, owing to other causes of liver injuries/pathologies. However, to date, no research has been carried out detailing clinical impact of minimal-mild degrees of steatosis upon healthy subjects. Participants practising unhealthy diet were affected significantly from steatosis. Diabetics and hypertensive subjects were specifically found with grade 1 steatosis. Liver enzymes were raised in moderate to severe cases only. Combination of liver enzymes and ultrasound could prove good in investigational planning, followed by
MR-scan in suspicious cases to rule out false positives. Thus it is a suitable modality for diagnosing moderate to severe fatty liver. It is most widely used in clinical practise today and will remain popular in future due to its widespread availibility and excellent tolerability.

We recommend to evaluate the diagnostic implications of expansions in ultrasound technology and of more comprehensive quantification of hepatic steatosis by imaging units with different field-strengths and frequencies. In addition, there is a need to investigate the cost-effectiveness of noninvasive evaluations in diagnosing NAFLD, tracking disease progression and monitoring responses to therapies.

\section{ACKNOWLEDGEMENTS}

We value and appreciate Mr. Asif Hanif (head Biostatistician), Ms Mehreen Fatima \& Mr. Kashif Siddique for statistical work analysis. Also to all the Medical Technologists at IDC, thanks for being generous during our data collection days there. Gratitude to Dr. Faiza Javed and Dr. Ayesha Ashfaq for being considerate towards our work. Special thanks to Ms.Saria Rajpoot (Sr.Radiologic Technologist) and Dr.Rustam Alam (Radiologist) for their generosity, dedication and help throughout the study. Ms. Shamsa for counselling participants and cooperation for anthropometric data acquisition. Heartious thanks to Mr.Quonnel Muhammad Siddique for granting us permission to collect research data at IDC.

This research received no specific grant from any funding agency in the public, commercial, or notfor-profit sectors.

\section{Supplemental Material}

Sample size was calculated with this formula
$=\frac{z_{1-\frac{\alpha}{2}}^{2} p q}{d^{2}}, \mathrm{p}=0.07 \quad[5,29], \mathrm{q}=0.93, \mathrm{z}=1.96, \mathrm{~d}=0.05$ Incidence is $7 \%$ and calculated sample size is 86 . Other data will be furnished upon demand.

\section{RECOMMENDATIONS}

A few studies have suggested that weight loss may be associated with regression of fat within the liver. Therefore, the most important recommendations for people with fatty liver are to lose weight if they are overweight or obese, increase their physical activity, follow a balanced diet and avoid alcohol and unnecessary medications. New evidence suggests that Mediterranean diet (rich in monounsaturated fatty acids) may be more beneficial than low fat diet. Drinking coffee seems to decrease the risk of having fatty liver in large cohort studies. In patients with $\mathrm{NASH}$, the more severe form of NAFLD, these same recommendations may be helpful. It is also important to control diabetes and treat elevated cholesterol levels when appropriate. Development of medications that could treat NAFLD and NASH is an area of intense 
research. Strategies currently being evaluated by physicians and scientists to decrease the amount of fat/ inflammation in the liver include:

- Weight reduction (diet + exercise, medications, surgery)

- $\quad$ Lipid lowering medications (e.g, N-3 fish oil)

- Insulin sensitizers (medications)

- Decrease the amount of liver inflammation by administering anti-oxidant medications, antiapoptotic medications and anti-cytokine medications

- Dietary nutritional management should be a component of any treatment plan for NAFLD.

Note: We donot recommend practising conventional ultrasound after therapeutic interventions for follow-up of patients with NAFLD. For this regard, MRI-PDFF or quantitative ultrasound is recommended.

\section{Source(s) of technical support: IDC}

Ethical Approval: Ethical committee at The University of Lahore -54000 .

Conflict of interest: None declared.

\section{REFERENCES}

1. Lupsor, M., \& Badea, R. (2005). Imaging diagnosis and quantification of hepatic steatosis: is it an accepted alternative to needle biopsy. Rom J Gastroenterol, 14(4), 419-425.

2. Vernon, G., Baranova, A., \& Younossi, Z. (2011). Systematic review: the epidemiology and natural history of non-alcoholic fatty liver disease and non-alcoholic steatohepatitis in adults. Alimentary pharmacology \& therapeutics, 34(3), 274-285.

3. Ma, X., Holalkere, N.-S., Mino-Kenudson, M., Hahn, P. F., \& Sahani, D. V. (2009). Imagingbased quantification of hepatic fat: methods and clinical applications. Radiographics, 29(5), 12531277.

4. Zois, C. D., Baltayiannis, G. H., Bekiari, A., Goussia, A., Karayiannis, P., Doukas, M., . . . Mitsi, V. (2010). Steatosis and steatohepatitis in postmortem material from Northwestern Greece. World journal of gastroenterology: WJG, 16(31), 3944.

5. Amarapurkar, D. N., Hashimoto, E., Lesmana, L. A., Sollano, J. D., Chen, P. J., Goh, K. L., \& NAFLD1, A. P. W. P. o. (2007). How common is non-alcoholic fatty liver disease in the AsiaPacific region and are there local differences? Journal of gastroenterology and hepatology, 22(6), 788-793.

6. Loomba, R., \& Sanyal, A. J. (2013). The global NAFLD epidemic. Nature reviews Gastroenterology \& hepatology, 10(11), 686-690.

7. Hamer, O. W., Aguirre, D. A., Casola, G., Lavine, J. E., Woenckhaus, M., \& Sirlin, C. B. (2006).
Fatty liver: imaging patterns and pitfalls. Radiographics, 26(6), 1637-1653.

8. Bohte, A. E., van Werven, J. R., Bipat, S., \& Stoker, J. (2011). The diagnostic accuracy of US, CT, MRI and $1 \mathrm{H}-\mathrm{MRS}$ for the evaluation of hepatic steatosis compared with liver biopsy: a meta-analysis. European radiology, 21(1), 87-97.

9. Caussy, C., Reeder, S. B., Sirlin, C. B., \& Loomba, R. (2018). Noninvasive, quantitative assessment of liver fat by MRI-PDFF as an endpoint in NASH trials. Hepatology, 68(2), 763-772.

10. Cheung, O., \& Sanyal, A. J. (2008). Hepatitis C infection and nonalcoholic fatty liver disease. Clinics in liver disease, 12(3), 573-585.

11. Pessayre, D., Berson, A., Fromenty, B., \& Mansouri, A. (2001). Mitochondria in steatohepatitis. Paper presented at the Seminars in liver disease.

12. Reid, A. E. (2001). Nonalcoholic steatohepatitis. Gastroenterology, 121(3), 710-723.

13. Tominaga, K., Kurata, J. H., Chen, Y. K., Fujimoto, E., Miyagawa, S., Abe, I., \& Kusano, Y. (1995). Prevalence of fatty liver in Japanese children and relationship to obesity. Digestive diseases and sciences, 40(9), 2002-2009.

14. Raptis, D. A., Fischer, M. A., Graf, R., Nanz, D., Weber, A., Moritz, W., . . Clavien, P.-A. (2012). MRI: the new reference standard in quantifying hepatic steatosis? Gut, 61(1), 117-127.

15. Ratziu, V., Charlotte, F., Heurtier, A., Gombert, S., Giral, P., Bruckert, E., . . . Group, L. S. (2005). Sampling variability of liver biopsy in nonalcoholic fatty liver disease. Gastroenterology, 128(7), 1898-1906.

16. Johnson, N. A., Sachinwalla, T., Walton, D. W., Smith, K., Armstrong, A., Thompson, M. W., \& George, J. (2009). Aerobic exercise training reduces hepatic and visceral lipids in obese individuals without weight loss. Hepatology, 50(4), 1105-1112.

17. Lee, S. S., \& Park, S. H. (2014). Radiologic evaluation of nonalcoholic fatty liver disease. World journal of gastroenterology: WJG, 20(23), 7392.

18. Meisamy, S., Hines, C. D., Hamilton, G., Sirlin, C. B., McKenzie, C. A., Yu, H., . . . Reeder, S. B. (2011). Quantification of hepatic steatosis with T1-independent, T2*-corrected MR imaging with spectral modeling of fat: blinded comparison with MR spectroscopy. Radiology, 258(3), 767-775.

19. Andronescu, C. I., Purcarea, M. R., \& Babes, P. A. (2018). The role of noninvasive tests and liver biopsy in the diagnosis of nonalcoholic fatty liver disease. Journal of medicine and life, 11(3), 243.

20. Hernaez, R., Lazo, M., Bonekamp, S., Kamel, I., Brancati, F. L., Guallar, E., \& Clark, J. M. (2011). Diagnostic accuracy and reliability of ultrasonography for the detection of fatty liver: a meta-analysis. Hepatology, 54(3), 1082-1090. 
21. Feldstein, A. E., \& Kay, M. H. (2012). Fatty liver disease. The American College of Gastroenterology. Diunduh dari: www. acg. gi. org. Tanggal, 19.

22. Mouzaki, M., \& Allard, J. P. (2012). The role of nutrients in the development, progression, and treatment of nonalcoholic fatty liver disease. Journal of clinical gastroenterology, 46(6), 457467.

23. Joseph, A. E. A., Saverymuttu, S. H., Al-Sam, S., Cook, M. G., \& Maxwell, J. D. (1991). Comparison of liver histology with ultrasonography in assessing diffuse parenchymal liver disease. Clinical Radiology, 43(1), 26-31. doi: 10.1016/S0009-9260(05)80350-2

24. Bedossa, P., Dargère, D., \& Paradis, V. (2003). Sampling variability of liver fibrosis in chronic hepatitis C. Hepatology, 38(6), 1449-1457.

25. Dasarathy, S., Dasarathy, J., Khiyami, A., Joseph, R., Lopez, R., \& McCullough, A. J. (2009). Validity of real time ultrasound in the diagnosis of hepatic steatosis: a prospective study. Journal of hepatology, 51(6), 1061-1067.

26. Yu, H., McKenzie, C. A., Shimakawa, A., Vu, A. T., Brau, A. C., Beatty, P. J., . . . Reeder, S. B. (2007). Multiecho reconstruction for simultaneous water-fat decomposition and $\mathrm{T} 2 *$ estimation. Journal of Magnetic Resonance Imaging: An Official Journal of the International Society for Magnetic Resonance in Medicine, 26(4), 11531161.

27. Kim, M., Kang, B.-K., \& Jun, D. W. (2018). Comparison of conventional sonographic signs and magnetic resonance imaging proton density fat fraction for assessment of hepatic steatosis. Scientific reports, 8(1), 7759.

28. Tang, A., Desai, A., Hamilton, G., Wolfson, T., Gamst, A., Lam, J., . . . Ang, B. D. (2014). Accuracy of MR imaging-estimated proton density fat fraction for classification of dichotomized histologic steatosis grades in nonalcoholic fatty liver disease. Radiology, 274(2), 416-425.

29. Ismail, F. W., \& Hamid, S. (2004). Hepatic steatosis and hepatitis C.

30. Kapuria, D., Takyar, V. K., Etzion, O., Surana, P., O'Keefe, J. H., \& Koh, C. (2018). Association of hepatic steatosis with subclinical atherosclerosis: Systematic Review and meta-analysis. Hepatology communications, 2(8), 877-887.

31. Maor, Y., \& Malnick, S. (2013). Liver injury induced by anticancer chemotherapy and radiation therapy. International journal of hepatology, 2013.

32. Kromrey, M., Ittermann, T., Berning, M., Kolb, C., Hoffmann, R., Lerch, M., . . . Kühn, J.-P. (2019). Accuracy of ultrasonography in the assessment of liver fat compared with MRI. Clinical radiology, 74(7), 539-546.

33. Bohte, A. E., Koot, B. G., van der Baan-Slootweg, O. H., Rijcken, T. H. P., Van Werven, J. R., Bipat, S., . . . Stoker, J. (2012). US cannot be used to predict the presence or severity of hepatic steatosis in severely obese adolescents. Radiology, 262(1), 327-334.

34. Kühn, J.-P., Hernando, D., Muñoz del Rio, A., Evert, M., Kannengiesser, S., Völzke, H., . . . Reeder, S. B. (2012). Effect of multipeak spectral modeling of fat for liver iron and fat quantification: correlation of biopsy with MR imaging results. Radiology, 265(1), 133-142.

35. Lin, S. C., Heba, E., Wolfson, T., Ang, B., Gamst, A., Han, A., . . S Sirlin, C. B. (2015). Noninvasive diagnosis of nonalcoholic fatty liver disease and quantification of liver fat using a new quantitative ultrasound technique. Clinical Gastroenterology and Hepatology, 13(7), 1337-1345. e1336.

36. Elliot, J. G., Donovan, G. M., Wang, K. C., Green, F. H., James, A. L., \& Noble, P. B. (2019). Fatty airways: implications for obstructive disease. European Respiratory Journal, 54(6). 